\title{
Morphological Analysis of Abursate Nematodes Parasitic in the Lungs of Mammals
}

\author{
Jessica Brenneman*, Ramon A. Carreno*, and Laura Tuhela* \\ * Department of Zoology, Ohio Wesleyan University, 61 S. Sandusky St., Delaware, OH 43015
}

Light and scanning electron microscopy (SEM) were used to study the morphology of two species of abursate lung worms that belong to a group of nematodes in which males of most species have a copulatory bursa. In abursate species, the bursa and its rays are replaced with simple papillae. Recent phylogenetic analyses have shown that the loss of the bursa evolved several times in the phylogeny of the lung nematodes from mammals [1]. However, the abursate nematodes have never been described beyond light microscopy observations. The two species observed here included Filaroides martis, a parasite common in the lungs of mink (Mustela vison) in North America, and Oslerus osleri, a nematode found in nodules in the trachea of coyotes (Canis latrans).

Using light microscopy, the overall structure of the male tail was distinct but the arrangement of papillae was difficult to observe (FIGS. 1, 2). Previous descriptions of the tail morphology of $F$. martis and $O$. osleri are based solely on light microscopy, resulting in vague drawings or inconsistent illustrations. For example, Anderson [2] shows F. martis with four papillae surrounding the cloaca along with two smaller papillae above the cloaca while Ko and Anderson [3] show $F$. martis with six papillae only surrounding the cloaca. Seneviratna shows $O$. osleri with seven papillae in a ring but the drawing does not show the precise relationship of these papillae with the cloaca [4].

To clarify the morphological description, the position of the tail papillae of $F$. martis and O. osleri was examined using SEM. Specimens were collected and preserved in 5\% formalin before being dehydrated in a graded ethanol series, critical point dried using an Autosamdri 795 Supercritical Point Dryer, and sputter coated with gold before imaging with a LEO 435VP SEM. In F. martis male specimens, five papillae surrounding the cloaca with two smaller papillae located directly above the cloaca was observed (FIG. 3). O. osleri males had six papillae surrounding the cloaca (FIG. 4).These patterns differ from previously published descriptions [2,3,4].

These SEM images not only clarify the morphology of the tail from abursate species but also demonstrate the importance of SEM for studying the morphology of nematodes. Further analysis is required to better understand how the morphology of abursate nematodes and bursate nematodes are related providing evolutionary information on the loss of the bursa in several lungworm clades.

\section{References}

[1] R.A. Carreno and S. A. Nadler. J. Parasitology. 89 (2003) 965.

[2] R. C. Anderson. Can. J. Zoology. 40 (1962) 893.

[3] R. C. Ko and R. C. Anderson. Can. J. Zoology. 50 (1972) 1555.

[4] P. Seneviratna. J. Helminthology. 33 (1959) 123. 


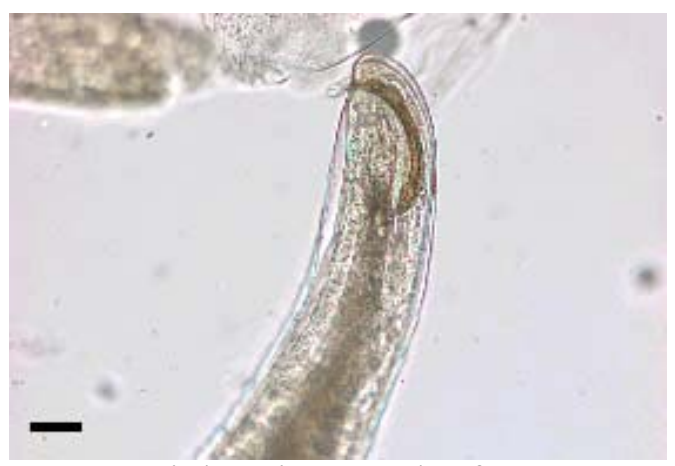

FIG. 1. Light micrograph of F. martis male tail. $(\mathrm{Bar}=45 \mu \mathrm{m})$

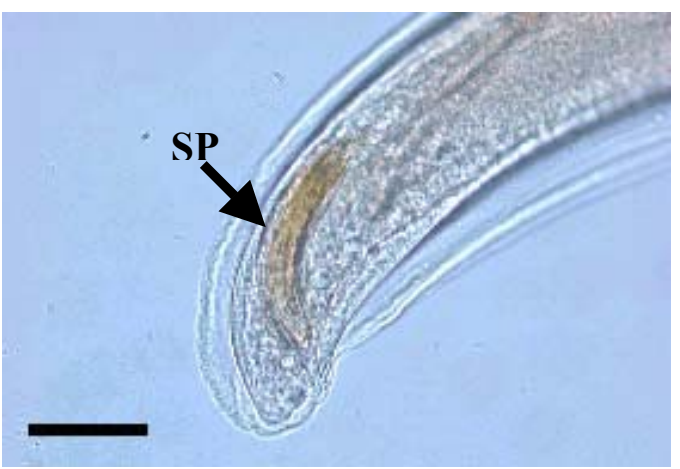

FIG. 2. Light micrograph of an O. osleri male tail showing papillae at the tip and the spicules (SP). $(\mathrm{Bar}=45 \mu \mathrm{m})$

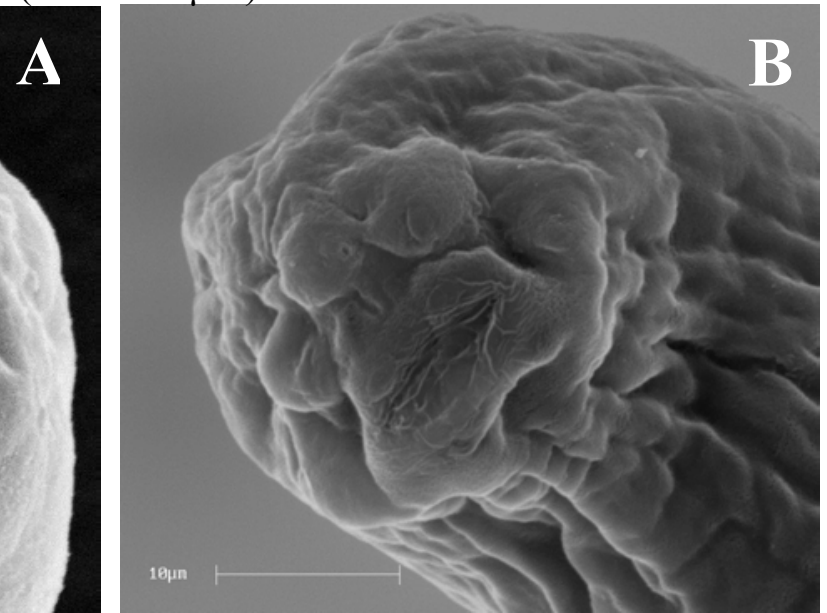

FIG. 3. SEM images of the F. martis male tail. A. Ventral view of the tail, with five papillae around the cloaca and two smaller papillae located directly anterior to the cloaca. B.Ventrolateral view showing the five papillae encircling the cloaca with two additional papillae located at the distal edge of the cloaca. $($ Bars $=10 \mu \mathrm{m})$

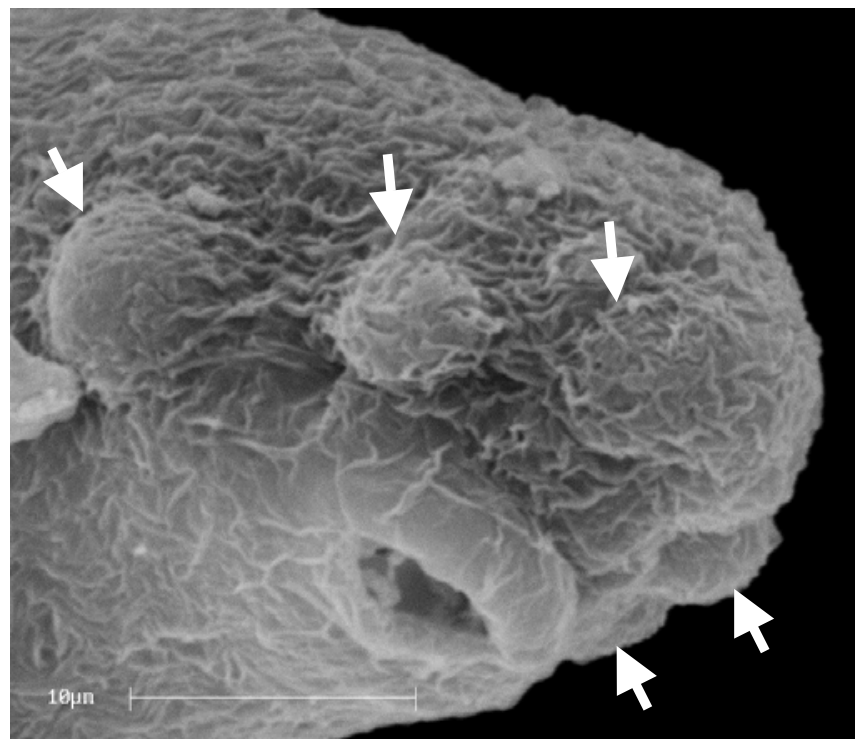

FIG. 4. Dorsolateral view of $O$. osleri male tail showing five of six papillae (arrows). $($ Bar $=10 \mu \mathrm{m})$ 